\title{
Discrete wavelet transform-based color image watermarking using uncorrelated color space and artificial bee colony
}

\author{
Manish Gupta ${ }^{1}$, Girish $\operatorname{Parmar}^{2}$, Rajeev Gupta $^{3}$,Mukesh Saraswat ${ }^{4}$ \\ ${ }^{1,2,3}$ Department of Electronics \& Communication Engineering, \\ University College of Engineering, \\ Rajasthan Technical University, Kota, India \\ ${ }^{1}$ E-mail: manish_dabra@yahoo.com \\ ${ }^{2}$ E-mail: girish_parmar2002@yahoo.com \\ ${ }^{3}$ E-mail: rajeev_eck@yahoo.com \\ ${ }^{4}$ Jaypee Institute of Information Technology, \\ Noida, India \\ E-mail:saraswatmukesh@gmail.com
}

Received 18 June 2014

Accepted 10 November 2014

\begin{abstract}
The exponential growth in electronic data over internet have increased the demand of a robust and high quality watermarking method for authentication and copyright protection. In general, the existing digital image watermarking methods embed the binary or gray scale watermark into the host image although most multimedia images are available in color. Moreover, available digital image watermarking methods generally use the correlated color spaces which impose the limitations to researchers for using only one color component at a time for embedding the watermark. Therefore, in this paper, a novel discrete wavelet transform (DWT) based color image watermarking method has been proposed which embeds the color watermark into host image using uncorrelated color space (UCS) and artificial bee colony (ABC) method. The results show that proposed method outperforms other existing methods against the various signal processing attacks.
\end{abstract}

Keywords: Image watermarking; Discrete wavelet transform; Uncorrelated color space; Artificial bee colony.

\section{Introduction}

During the recent years, the intensification of high data rate have discovered the new prospects of ecommerce such as electronically publishing, marketing, distribution, and transmission of real time multimedia data in the form of image, audio, video, etc. Although the digitization of multimedia contents have many advantages against their analog counterpart, the service providers are cautious to tender the services in their digital structure due to the unobstructed replication and broadcasting of copyrighted materials. In past decade, researchers proposed different methods for the protection of

\footnotetext{
*Department of Electronics \& Communication Engineering, University College of Engineering, Rajasthan Technical University, Kota, India
} 
multimedia data out of which digital watermarking methods outperform the other methods of protection such as cryptography, encryption, steganography, etc. This paper focuses on the protection of color images from its unauthorized utilization using a novel digital color image watermarking method.

Digital image watermarking methods can be categorized into spatial domain and transform domain watermarking. Spatial domain watermarking methods embed the watermark into the host image in their spatial co-ordinates ${ }^{12}$ while in transform-domain ${ }^{3}$, watermarks are embedded in certain transform domain such as discrete frequency transform (DFT) ${ }^{4}$, discrete cosine transform (DCT) ${ }^{5}$, and discrete wavelet transform (DWT) 6789 . There are mainly two objectives of digital image watermarking methods namely; quality and robustness ${ }^{10}$. Researchers suggested that DWT-based methods of image watermarking are more robust against the common signal processing and malicious attacks ${ }^{11} 1213$.

DWT-based image watermarking methods are capable to embed a fairly good quality of watermark and it can recover the watermark from watermarked image effectively. The quality and robustness of DWT-based methods depend on the selection of particular filter bank and decomposition level. Agreste and Andaloro ${ }^{14}$ developed a DWT-based method for any size of image which implants watermark data into high-frequency sub-bands of DWT coefficient. This watermarked data is imperceptible as per human visual system (HVS) directions. Later, Agreste and Andaloro ${ }^{15}$ modified the previous method by changing the filer bank by Daubechies- 2 and observed that it is more robust to geometric, filtering, and StirMark attacks with a low rate of false alarm. Ghouti et al. ${ }^{8}$ selected balanced multi-wavelets for the data hiding and found that the method is more robust against the standard watermarking attacks. Vahedi et al. ${ }^{9}$ exploited the advantage of symlet 4 filter bank to increase the quality and robustness of watermarking method as compared to existing methods. In his paper, they proposed a novel DWT-based method for color images by embedding the binary watermark. For embedding the watermark, DWT-based methods use three or higher level decompositions 679 . Vahedi et al. ${ }^{9}$ showed that three level decomposition with all the four subspaces namely; approximation, horizontal, vertical, and diagonal along with symlet-4 filter bank provide better results for image watermarking. Therefore, in this paper DWT has been used with three level of decomposition and symlet-4 filter bank for embedding the watermark.

Moreover, to increase the quality and robustness of watermarking methods, researchers also used many optimization methods like genetic algorithm (GA), particle swarm optimization (PSO), artificial bee colony (ABC), etc. $9 \begin{array}{llllll}16 & 17 & 18 & 19\end{array}$. PSO and GA have been used by Vahedi et al. ${ }^{9}{ }^{20}$ to calculate the optimized strength of watermark which improves the robustness of existing methods. Recently, Karaboga and Akay ${ }^{19}$ compared ABC with other optimization methods and concluded that the performance of $\mathrm{ABC}$ is better than or similar to other population-based optimization methods with the advantage of employing fewer control parameters. Therefore, in the present attempt $\mathrm{ABC}$ has been used to optimize the strength of the watermark.

Initially, image watermarking methods hide the watermark message into gray scale or color host image in the form of bits or bit stream, and later these digital bit streams are replaced by some pictorial shape like image ${ }^{67}$. Generally, various watermarking methods embed the gray scale or binary image watermark while very few work has been reported for color watermark 212223 , though most multimedia images are available in color. Generally, the researchers used RGB, $Y C_{b} C_{r}$, YIQ, HSI, HSV, etc. color space models for both watermarks and host images in their digital watermarking method. Golea et al. ${ }^{22}$ proposed the SVD-based RGB color image watermarking for embedding the color RGB watermark into the RGB host image. Recently, Su et al. ${ }^{23}$ presented $\mathrm{QR}$ decomposition method for embedding the RGB color watermark into RGB host image. In his paper, they concluded that the proposed method is robust against the attacks such as compression, filtering, cropping, etc. The above mentioned color models are correlated i.e. the image components are not independent and change in one component may affect the other components of the image. This imposes the constraints for the researchers to use only 
one color component at a time for embedding the watermark data. However, there exist some uncorrelated color models such as $L a b, L \alpha \beta$, etc. 2425 ${ }^{26}$ which may be used in color image watermarking to increase the robustness and quality by using all the color image components of host and watermark images. Chou and $\mathrm{Wu}^{21}$ embedded the color watermark in Lab color space using less computationally complex spatial-domain color image watermarking method. However, the robustness of this method is poor.

Therefore, due to the limitations of correlated color spaces, rare use of colored watermark images, and powerfulness of DWT, this paper proposes a novel DWT-based color image watermarking method using uncorrelated color space (UCS). Further, ABC has been used to optimize the strength factor of the proposed watermarking method.

Rest of the paper is organized as follows. Section 2 describes the working of DWT, UCS, and ABC methods used in this paper. The proposed method is presented in Section 3. Section 4 describes the method validation parameters and experimental results are discussed in Section 5. Finally, Section 6 concludes the paper.

\section{Preliminaries}

\subsection{Discrete Wavelet Transform (DWT)}

DWT is a sampled version of continuous wavelet transform. The main advantage of DWT is that it maintains both frequency and time information at the same time which was missing in DFT. DWT is used to decompose the input image into sub-images of diverse spatial domain and independent frequency area ${ }^{7} 8$. Transformation of an image from the spatial domain to DWT domain by one level decomposes the input image into four different frequency bands in which one is the low frequency and remaining three are the high frequency bands and represented as LL (approximation detail of image), HL (horizontal detail of image), LH (vertical detail of image), and $\mathrm{HH}$ (diagonal detail of image) respectively. After one level decomposition, the further decomposition of given image is done using only LL sub-space which is also decomposed into four dis- tinct frequency bands as mention above. Figure 1 shows the two dimensional image of size $512 \times 512$ before and after the three level of DWT decomposition with their sub-spaces size.

To embed the watermark in DWT-based decomposed image, the transform coefficients of DWT are modified by watermark. Since, the low frequency band (LL) of DWT decomposed image is similar to the original image, most of the information or energy of original image lies in this frequency band.

\subsection{Uncorrelated Color Space (UCS)}

The quality of the color image watermarking methods depends on how images are split into three color channels, i.e. which color space was chosen. For better quality, color space must be uncorrelated which makes the three color channels semiindependent 2728 and may be used for embedding the watermark. This paper uses the recently developed UCS proposed by Liu ${ }^{29}$. UCS is derived from RGB color space using principal component analysis (PCA). UCS uses a linear transformation, $W_{U} \in \mathbb{R}^{3 \times 3}$, of the RGB color space to uncorrelate the component images as shown in Eq. (1) ${ }^{29}$;

$$
\left[\begin{array}{l}
U(x, y) \\
C(x, y) \\
S(x, y)
\end{array}\right]=W_{U}\left[\begin{array}{l}
R(x, y) \\
G(x, y) \\
B(x, y)
\end{array}\right]
$$

The $W_{U}$ is calculated by factorizing the covariance matrix $\mathrm{C}$ using PCA in the following form ${ }^{29}$ :

$$
C=W_{U}^{t} \Lambda W_{U}
$$

here $W_{U}^{t}$ and $\Lambda$ are the orthogonal eigenvector matrix and diagonal eigenvalue matrix with diagonal elements in a decreasing order, respectively. Saraswat and Arya ${ }^{28}$ used UCS for color transfer of images and observed that UCS outperforms the other uncorrelated color spaces.

\subsection{Artificial Bee Colony $(A B C)$ Method}

ABC ${ }^{19}$ is a newly developed swarm intelligencebased method which is inspired by the intelligent food foraging behavior of honey bees. Similar to the other population-based methods, ABC solution 
512

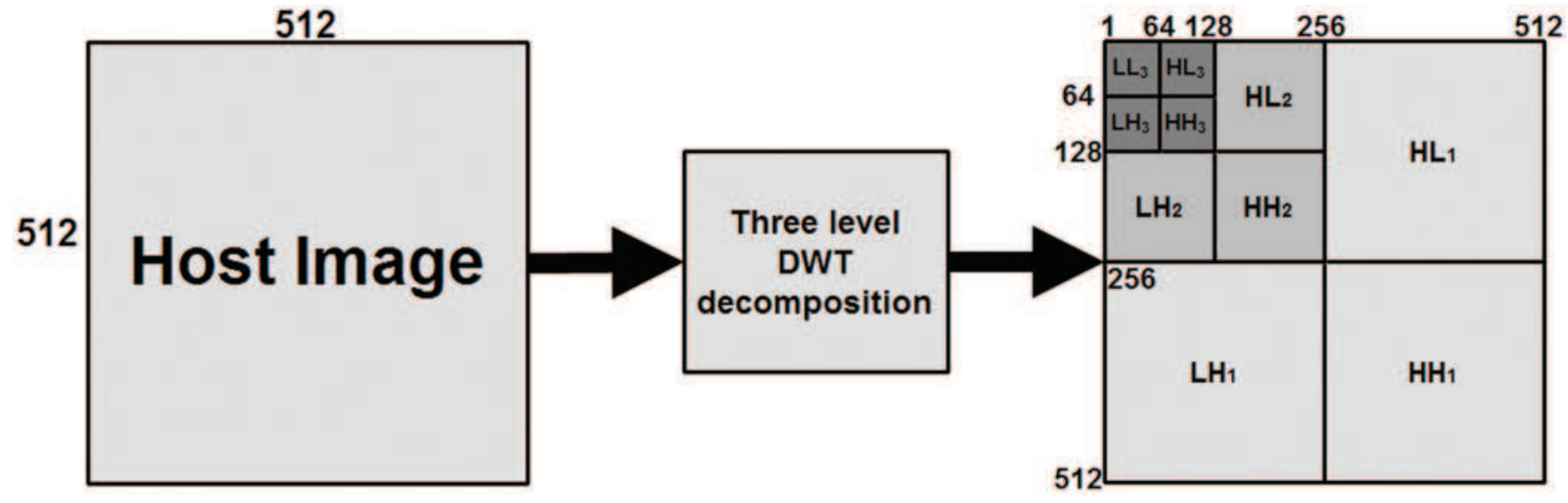

Figure 1: Three level decomposition layout of an image.

search process is an iterative process. After, initialization of the $\mathrm{ABC}$ parameters and swarm, it requires the repetitive iteration of the three phases namely; employed bee phase, onlooker bee phase, and scout bee phase. The initialization of the swarm and details of each phase are described in the following sections.

\subsubsection{Initialization of the Swarm}

The parameters for ABC are the number of food sources, number trials after which a food source is considered to be abandoned, and termination criteria. In the basic $\mathrm{ABC}$, the number of food sources is equal to the employed bees or onlooker bees. Initially, a uniformly distributed initial swarm of $S N$ food sources, where each food source $x_{i}(i=$ $1,2, \ldots, S N)$ is a $D$-dimensional vector, are generated. Here $D$ is the number of variables in the optimization problem and $x_{i}$ represent the $i^{t h}$ food source in the swarm. Each food source is generated as mentioned in Eq. (3) ${ }^{19}$

$$
x_{i j}=x_{\min j}+\operatorname{rand}[0,1]\left(x_{\operatorname{maxj}}-x_{\min j}\right)
$$

here, $x_{\min j}$ and $x_{\operatorname{maxj}}$ are bound of $x_{i}$ in $j^{\text {th }}$ direction and $\operatorname{rand}[0,1]$ is a uniformly distributed random number in the range $[0,1]$.

\subsubsection{Employed Bee Phase}

In employed bee phase, employed bees modify the current solution (food source) based on the informa- tion of individual experience and the fitness value of the new solution. If the fitness value of the new solution is higher than that of the old solution, the bee updates her position with the new one and discards the old one. The position update equation for $i^{\text {th }}$ candidate in this phase is presented in Eq. (4) ${ }^{19}$.

$$
v_{i j}=x_{i j}+\phi_{i j}\left(x_{i j}-x_{k j}\right)
$$

here, $k \in\{1,2, \ldots, S N\}$ and $j \in\{1,2, \ldots, D\}$ are randomly chosen indices. $k$ must be different from $i$. $\phi_{i j}$ is a random number between $[-1,1]$.

\subsubsection{Onlooker Bees Phase}

After completion of the employed bees phase, the onlooker bees phase starts. In onlooker bees phase, all the employed bees share the new fitness information (nectar) of the new solutions (food sources) and their position information with the onlooker bees in the hive. Onlooker bees analyze the available information and select a solution with a probability, $\operatorname{prob}_{i}$, related to its fitness. The probability $\operatorname{prob}_{i}$ may be calculated using Eq. (5) ${ }^{19}$.

$$
\text { prob }_{i}=\frac{\text { fitness }_{i}}{\sum_{i=1}^{S N} \text { fitness }_{i}}
$$

here, fitness is $_{i}$ is the fitness value of the solution $i$. As in the case of the employed bee, it produces a modification on the position in its memory and checks the fitness of the candidate source. If the fitness is higher than that of the previous one, the bee memorizes the new position and forgets the old one. 


\subsubsection{Scout Bees Phase}

If the position of a food source is not updated up to predetermined number of cycles, then the food source is assumed to be abandoned and scout bees phase starts. In this phase, the bee associated with the abandoned food source becomes scout bee and the food source is replaced by a randomly chosen food source within the search space. In $\mathrm{ABC}$, predetermined number of cycles is a crucial control parameter which is called limit for abandonment. Assume that the abandoned source is $x_{i}$. The scout bee replaces this food source by a randomly chosen food source which is generated as given in Eq. (6) ${ }^{19}$.

$x_{i}^{j}=x_{\text {min }}^{j}+\operatorname{rand}[0,1]\left(x_{\text {max }}^{j}-x_{\text {min }}^{j}\right), \quad$ for $j \in\{1,2, \ldots, D\}$

here, $x_{\min j}$ and $x_{\operatorname{maxj}}$ are bound of $x_{i}$ in $j^{\text {th }}$ direction. The pseudo-code of the $\mathrm{ABC}$ is shown in following algorithm ${ }^{19}$. In this paper, $A B C$ is used to optimize the watermarking parameters for increasing the quality and robustness of the proposed method.

\section{Artificial Bee Colony Algorithm}

Initialize the parameters.

While(Termination criteria is not satisfied)

1. Employed bee phase for generating new food sources.

2. Onlooker bees phase for updating the food sources depending on their nectar amounts.

3. Scout bee phase for discovering the new food sources in place of abandoned food sources.

4. Memorize the best food source found so far.

5. If a termination criteria is not satisfied, go to step 1; otherwise output the best solution found so far.

\section{End While}

\section{Proposed Methodology}

The proposed method explores the advantages of uncorrelated color space over the correlated color space to improve the performance of watermarking methods in terms of quality and robustness. It implants the color watermark image into the color host image by modifying the decomposed wavelet coefficients of host image. The each channel of color image is embedded in the corresponding channel of host image to increase the reliability during the recovery process and protect against the common signal processing attacks. The proposed method consists of five phases namely; pre-processing of host and watermark image, watermark embedding, image postprocessing, extraction of watermark, and performance improvement using ABC. The basic structural design of the proposed method is shown in Figure 2. Since each color image has three channels, the structural design and methodology represented in Figure 2 are repeated for all the three channels. The details of each phase of the proposed method is described in the following sections.

\subsection{Image Pre-processing}

In image pre-processing, both the host $R G B$ color image $(H)$ and watermark image $(W)$ are transformed into UCS color space using Eq. (1) which produces six independent image components (three for host and three for watermark) namely; $H_{U}, H_{C}$, and $H_{S}$ for host image and $W_{U}, W_{C}$, and $W_{S}$ for watermark image. After transformation, each component of watermark image is divided into 16 non-overlapping sub-areas as shown in Figure 3. These sub-areas of watermark image are then re-arranged or scrambled by some pre-defined sequence or key to introduce one more level of security and enforced the user to use the key for the extraction of the image from watermarked image.

Further, third level of decomposition is applied on each component of host image using symlet-4 wavelet function. The resultant third level wavelet coefficients, $\left(H_{k 3}, k=\{L L, H L, L H, H H\}\right)$ is chosen for embedding process.

\subsection{Watermark Embedding}

After pre-processing of the images, the scrambled watermark is embedded into the DWT coefficients of host image. The third level decomposition coefficients of host image are $H_{L L 3}(x, y), H_{H L 3}(x, y), H_{L H 3}(x, y)$, and $H_{H H 3}(x, y)$ representing the approximation, horizontal, vertical, and diagonal details of 3-DWT decomposed host image respectively. In order to increase the reliability and robustness of proposed method against the malicious attacks, it is desired to hide each color component of the watermark image in corresponding component of host coefficients. Therefore, approximation details of the third level coefficients are divided into 16 non-overlapping areas as depicted in Figure 4. 


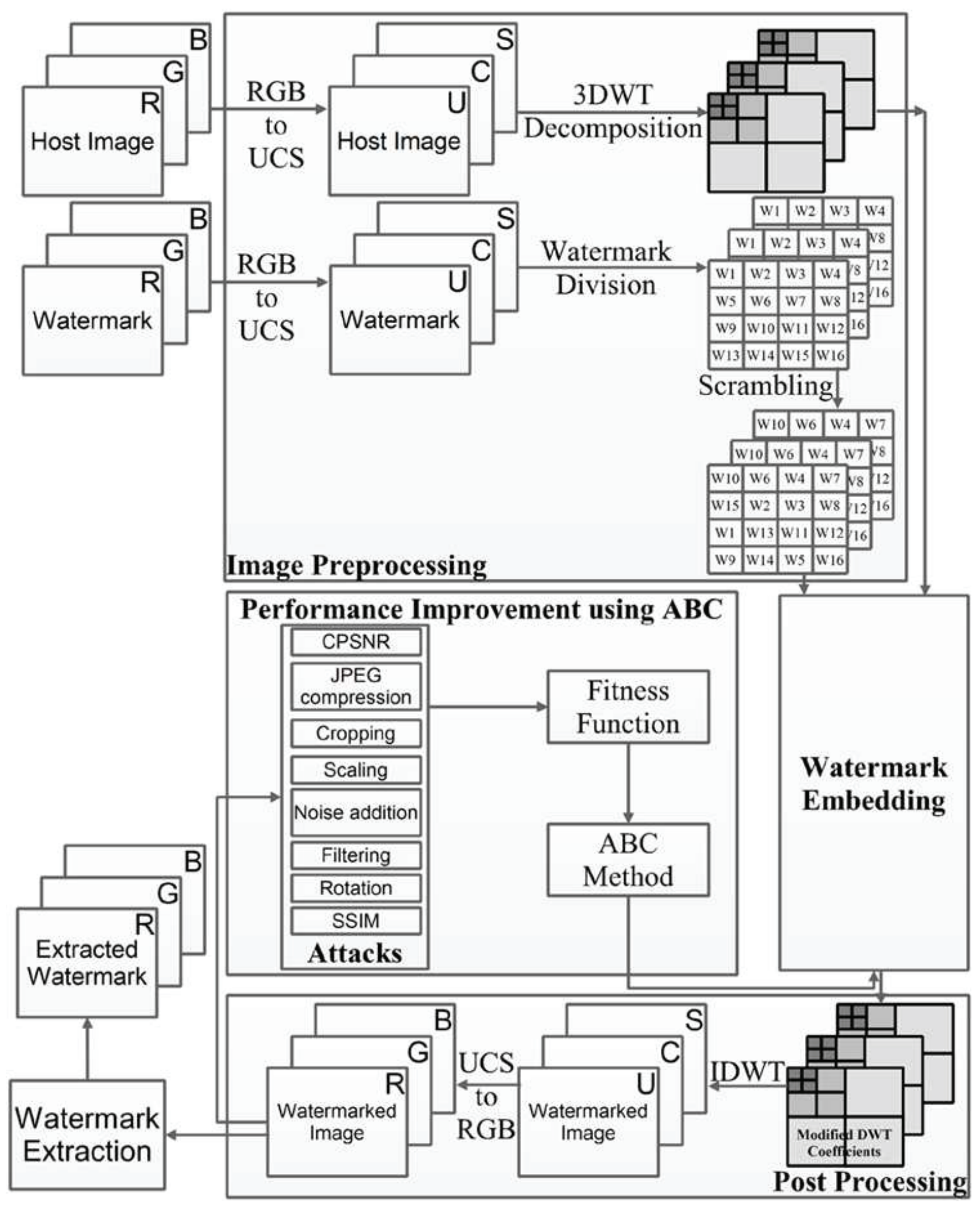

Figure 2: Structural design of proposed method. 


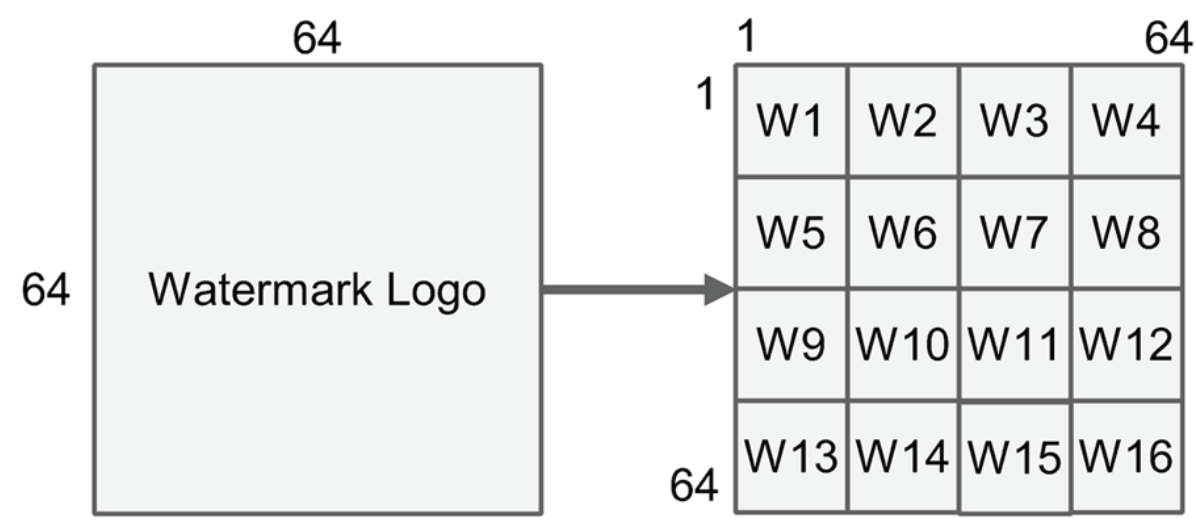

Figure 3: Watermark division.

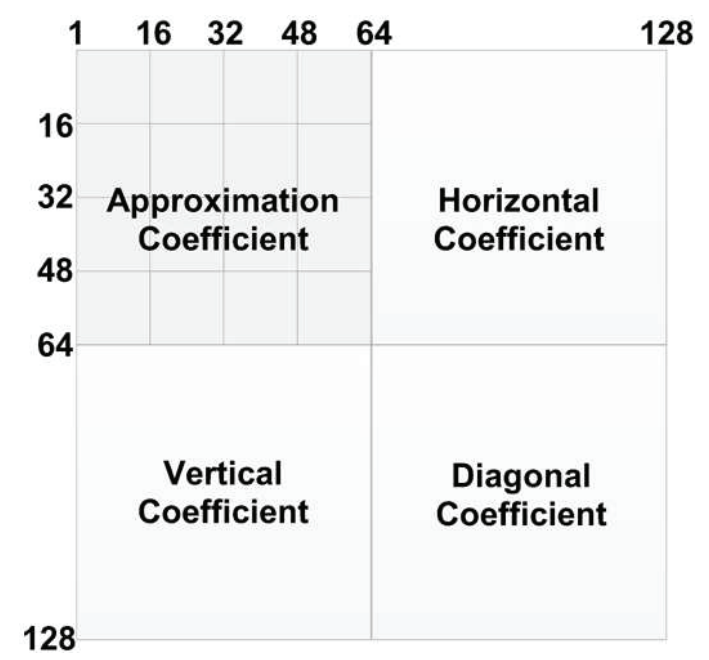

Figure 4: Partitioning of 3-DWT coefficients.

Now, the scrambled watermark is embedded into approximation details of the third level decomposed host image coefficients by using Eq. (7).

$$
\begin{gathered}
H W_{k 3}(x, y)=H_{k 3}(x, y)+\alpha(r) W_{p}(x, y) \\
k=\{L L, H L, L H, H H\} \\
p=\{1,2,3, \ldots, 16\} \\
r=\{1,2,3, \ldots, 16\}
\end{gathered}
$$

here, $k$ represents the sub-areas of host image, $p$ is the intended block of watermark, and $\alpha$ denotes the strength of modification done in the host coefficients.

\subsection{Post-processing}

After embedding all the color channels of UCS watermark image into corresponding DWT host image coef- ficients, inverse DWT (IDWT) is taken for watermarked host image coefficients which returns watermarked image in UCS color space. Finally, UCS watermarked image is reconverted into RGB color space.

\subsection{Watermark Extraction}

The watermark extraction process requires watermarked image and pre-defined key. To extract the watermark, RGB watermarked image is transformed into UCS color space and then 3-DWT decomposition using symlet-4 wavelet filter bank on each channel is applied. To gather the fraction watermarked image, a reverse process of embedding ia applied as shown in Figure 2 and using Eq. (8). Finally, the scrambled watermark image is rearranged to its original sequence.

$$
\begin{gathered}
\widehat{W}_{p}(x, y)=\frac{H_{k 3}(x, y)-H W_{k 3}(x, y)}{\alpha(r)} \\
k=\{L L, H L, L H, H H\} \\
p=\{1,2,3, \ldots, 16\} \\
r=\{1,2,3, \ldots, 16\}
\end{gathered}
$$

\subsection{Performance Improvement Using ABC Method}

The watermarked image $H W_{k 3}$ is subjected to various watermarking attacks which degrade the performance of watermarking methods. The design of the apposite attacks are as important as to design a method for protection of multimedia contents because they require to test the robustness and security of the newly design methods for protection of multimedia contents. There are mainly two parameters namely quality and robustness which must be maximized for a watermarking method, but these parameters are inversely related with each other i.e. if quality 
increases, robustness suffers and vice-versa. In this paper, optimum values of these parameters are dependent on the suitable values of strength factor $(\alpha)$. Therefore, this paper uses $\mathrm{ABC}$ optimization method for selecting the values of $\alpha$ which optimizes the quality and robustness in terms of fitness function.

There are 16 strength factors used in this paper which becomes the dimension of each individual in ABC method having the population size 50 . The optimized values of these 16 strength factors are calculated by minimizing the following fitness function using $\mathrm{ABC}$, which is the modified version of Vahedi et al. ${ }^{9}$,

$$
\begin{aligned}
\text { Fitness } & =\frac{100}{\operatorname{CPSNR}}+\frac{100}{\operatorname{SSIM}}+2 \times \sum_{Q=60 \%}^{90 \%}\left[1-N C_{j p g}(Q)\right] \\
& +\sum_{Q=10 \%}^{50 \%}\left[1-N C_{j p g(Q)}\right]+\sum_{i=1}^{5}\left[1-N C_{\text {filter }(i)}\right] \\
& +\sum_{i=1}^{3}\left[1-N C_{\text {scale }(i)}\right]+\sum_{i=1}^{3}\left[1-N C_{\text {noise }(i)}\right] \\
& +\sum_{i=1}^{4}\left[1-N C_{\text {crop }(i)}\right]+\left[1-N C_{\text {rotation }}\right]
\end{aligned}
$$

here, CPSNR is composite peak signal-to-noise ratio, SSIM is structural similarity, and NC is normalized correlation calculated for the attacks namely JPEG compression (10 to $90 \%)$, filtering (wiener, mean, median), scaling (0.6 to 1.2 factor), noise addition (poisson, salt and pepper, gaussian), rotation $\left(1^{0}\right)$, and cropping (10 to 40 $\%)$. These values are calculated by applying the mentioned attacks on watermarked image. The setting of the parameters for this experiment are given below:

- Colony size $N P=50$.

- $\phi_{i j}=\operatorname{rand}[-1,1]$.

- Number of food sources $S N=N P / 2$.

- limit $=1500$.

- The stopping criteria is maximum number of function evaluations (which is set to be 500) is reached.

\section{Method Validation}

The results obtained from the proposed method are compared with the methods of Chou and $\mathrm{Wu}^{21}$ and $\mathrm{Su}$ et al. ${ }^{23}$ who also embedded the color watermark images into the color host images. To compare these methods, quality and robustness parameters are considered. The quality of watermarked image can be assess by two ways namely; subjective and objective tests. Subjective test is done by 10 humans beings on the scale of 0 (very poor) to 5 (excellent) while objective test calculates the parameters namely; CPSNR and SSIM. CPSNR measures the degree of similarity between the original and watermarked image and is calculated as follows ${ }^{9}$ :

$C P S N R=\frac{10}{3} \sum_{s} \log \frac{M \times N \times 255^{2}}{\sum_{m=1}^{M} \sum_{n=1}^{N}[H(m, n, s)-H W(m, n, s)]^{2}}$

SSIM also measures the degree of similarity by including the three aspects of $H V S$ namely; loss of correlation (c), luminance distortion (1), and contrast distortion (s). It is formulated as follows ${ }^{23}$ :

$$
S S I M=l(H, H W) c(H, H W) s(H, H W)
$$

where,

$$
\begin{aligned}
l(H, H W) & =\frac{2 \mu_{H} \mu_{H W}+C_{1}}{\mu_{H}^{2}+\mu_{H W}^{2}+C_{1}} \\
c(H, H W) & =\frac{2 \sigma_{H} \sigma_{H W}+C_{2}}{\sigma_{H}^{2}+\sigma_{H W}^{2}+C_{2}} \\
s(H, H W) & =\frac{\sigma_{H H W}+C_{3}}{\sigma_{H} \sigma_{H W}+C_{3}}
\end{aligned}
$$

here $\mu$ and $\sigma$ are mean and standard deviation respectively while $C_{1}, C_{2}$, and $C_{3}$ are three positive constants used to avoid a null denominator. The values of CPSNR and SSIM must be maximized for effective quality of watermarked image.

The robustness of watermarking method shows the degree of similarity between the original watermark image and extracted watermark image after applying the attacks and is measured by normalized correlation (NC) which is formulated as follows:

$$
N C=\frac{1}{3} \sum_{s} \frac{\sum_{m=1}^{L} \sum_{n=1}^{L}[W(m, n, s) \times \widehat{W}(m, n, s)]}{\sum_{m=1}^{L} \sum_{n=1}^{L} W^{2}(m, n, s)}
$$

The values of NC lie in the rage of 0 (no similarity) to 1 (similar) and are calculated for the extracted watermarks without attack and with attacks.

\section{Experimental Results}

To compare the performance of proposed and considered color image watermarking methods, four popularly used 24-bit RGB color images namely; lena, mandrill, pepper, and sailboat, each of size $512 \times 512$, are used as host images which are shown in Figure 5. In this paper, one RGB color logo $(64 \times 64)$ and one RGB color image $(64 \times 64)$ namely; RTU logo and aeroplane color image respectively, are used as watermark images which are shown in 


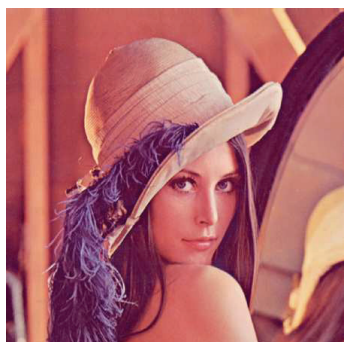

(a) Lena

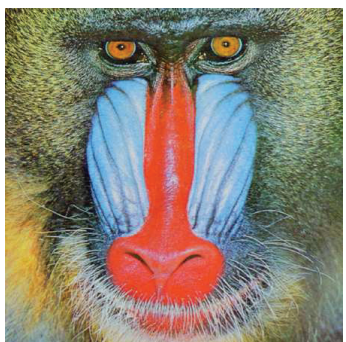

(b) Mandrill

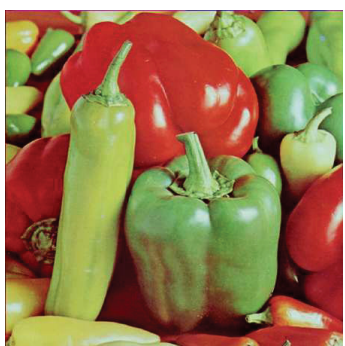

(c) Pepper

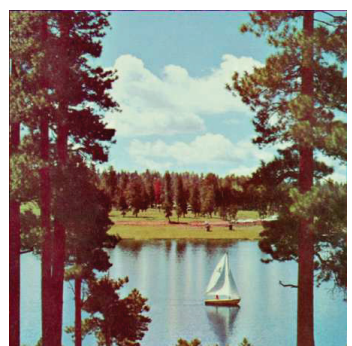

(d) Sailboat

Figure 5: RGB host images.

Figure 6. All the considered images have been taken from USC-SIPI image database ${ }^{30}$ except RTU logo which is taken from Rajasthan Technical University, Kota, India.

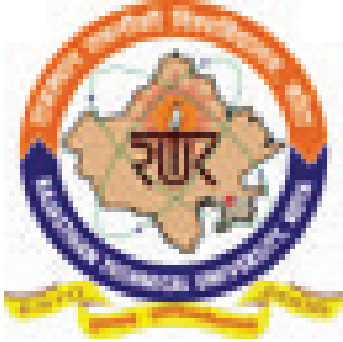

(a) RTU logo

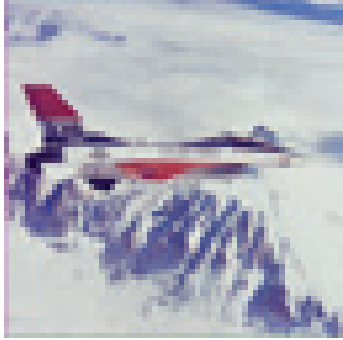

(b) Aeroplane
Figure 6: RGB watermark images.

In the pre-processing step of the proposed method, each host and watermark images are converted into UCS color space followed by third level decomposition of host images and scrambling of watermark images. Figure 7 shows the converted UCS images of host and watermark and third level decomposed lena host image is represented in Figure 8. The watermark image goes through the process of scrambling. One of the possible scrambled watermark images of RTU logo and aeroplane are shown in Figure 9.

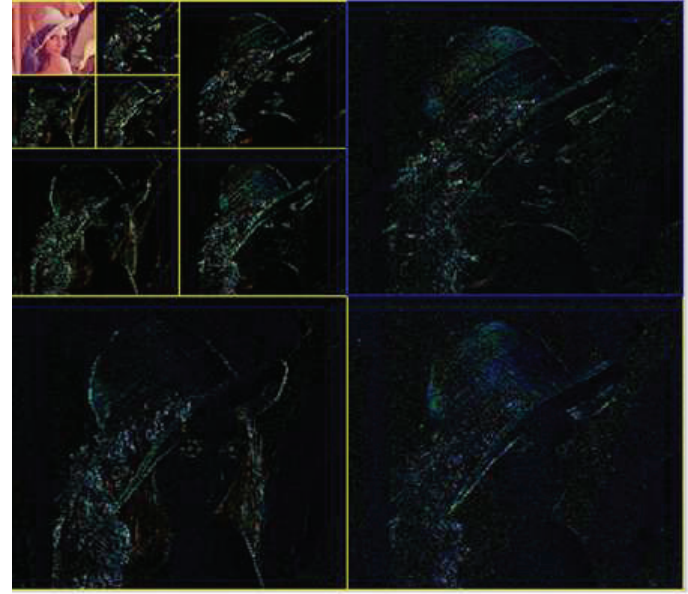

Figure 8: Three level decomposed host lena image using DWT.

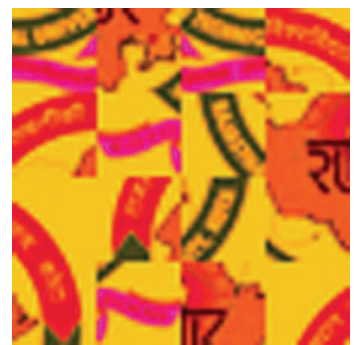

(a) RTU logo

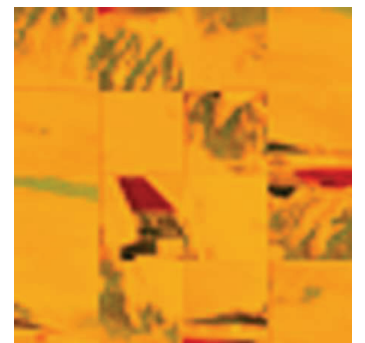

(b) Aeroplane
Figure 9: Representative scrambled UCS watermark images.

Now, the watermark images are embedded into each of the considered host images and the resultant watermarked images are shown in Figure 10. 


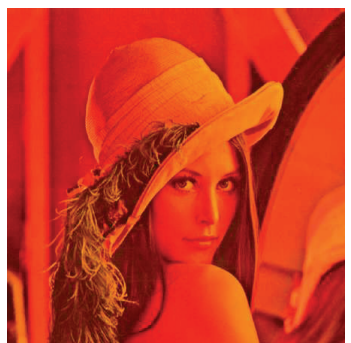

(a)

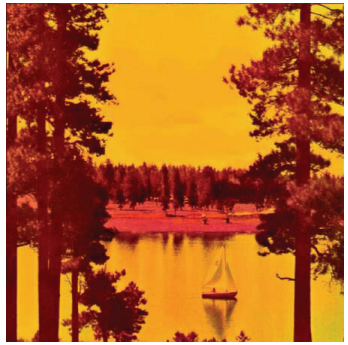

(d)

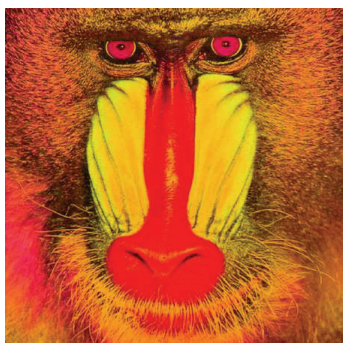

(b)

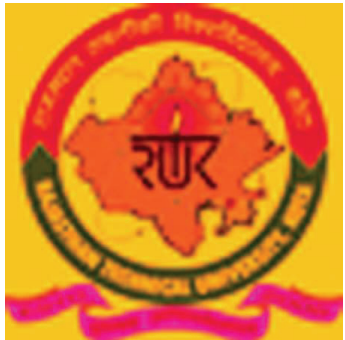

(e)

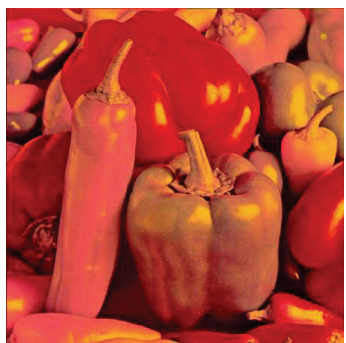

(c)

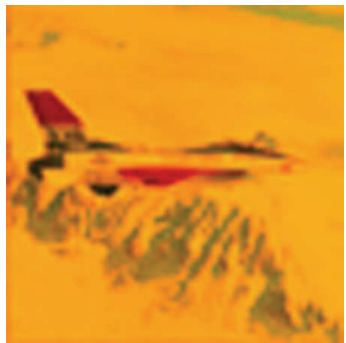

(f)

Figure 7: UCS host and watermark images (a). Lena, (b). Mandrill, (c). Pepper, (d). Sailboat, (e). RTU Logo, and (f). Aeroplane.

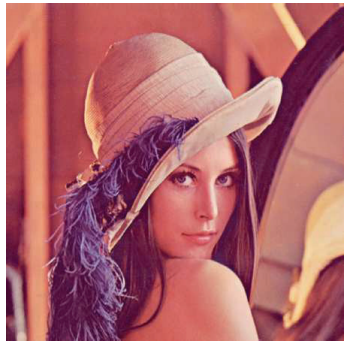

(a)

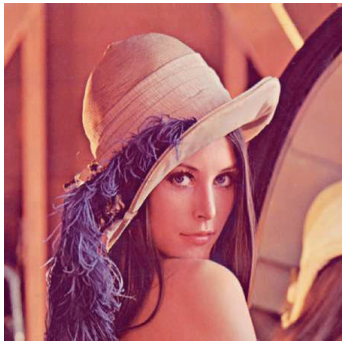

(e)

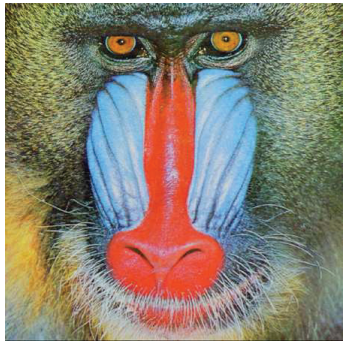

(b)

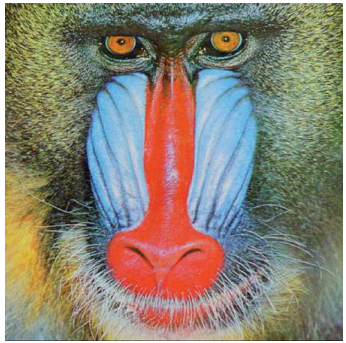

(f)

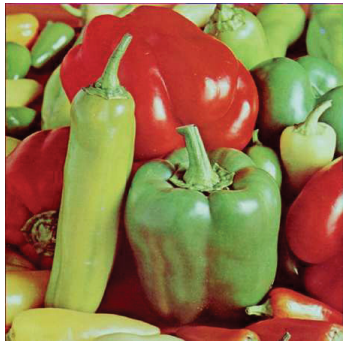

(c)

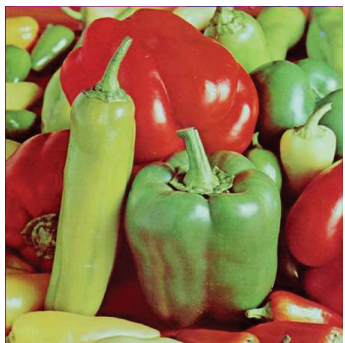

$(\mathrm{g})$

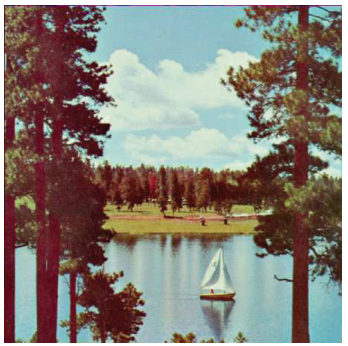

(d)

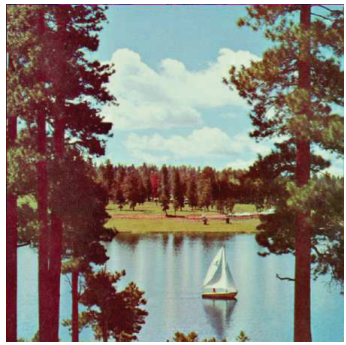

(h)

Figure 10: RGB watermarked images embedded by (a)-(d) RTU logo and (e)-(h) Aeroplane image 
Table 1: Comparison of CPSNR and SSIM values of watermarked images resultant from proposed and considered methods.

\begin{tabular}{|c|c|c|c|c|c|}
\hline S.No. & $\begin{array}{l}\text { Quality } \\
\text { Parameters }\end{array}$ & $\begin{array}{l}\text { Watermarked im- } \\
\text { ages }\end{array}$ & Su et al. ${ }^{23}$ & $\begin{array}{l}\text { Chou and Wu } \\
21\end{array}$ & $\begin{array}{l}\text { Proposed } \\
\text { Method }\end{array}$ \\
\hline \multirow[t]{4}{*}{1.} & CPSNR & Lena & 36.57 & 37.79 & 35.92 \\
\hline & & Mandrill & 36.42 & 37.71 & 35.67 \\
\hline & & Pepper & 36.61 & 37.01 & 35.23 \\
\hline & & Sailboat & 36.52 & 37.32 & 35.06 \\
\hline \multirow[t]{4}{*}{2.} & SSIM & Lena & 0.98 & 0.98 & 0.98 \\
\hline & & Mandrill & 0.98 & 0.98 & 0.96 \\
\hline & & Pepper & 0.96 & 0.97 & 0.96 \\
\hline & & Sailboat & 0.98 & 0.97 & 0.97 \\
\hline
\end{tabular}

To compare the objective test for quality measures of resultant watermarked images using proposed and considered methods, CPSNR and SSIM performance parameters are calculated and shown in Table 1. From Table 1 , it is validated that all the methods including proposed method maintain the quality of the watermarked image in terms of CPSNR $(>35 d B)$ and SSIM $(>0.96)$. However, the proposed method shows lower values of CPSNR and SSIM as compared to other considered methods due to the hiding of complete watermark into all three channels of host image while existing methods hide in only one channel of host image. Moreover, subjective test on the resultant watermarked images is performed and presented in Table 2. The results of subjective test show that the proposed method effectively embeds the watermarks which is imperceptible by human beings.

To show the effectiveness of the proposed watermarking method, the extracted watermarks from the watermarked images and their NC values are shown in Figure 11 for each of the considered method. From Figure 11 , it is visualized that the proposed method and Su et al. ${ }^{23}$ have highest $\mathrm{NC}$ values (1.0) for all extracted watermarks and hence outperforms the method of Chou and $\mathrm{Wu}{ }^{21}$. The columns of Figure 11 show extracted watermarks from considered watermarked images and first three rows show the extraction of RTU logo while last three show extraction of aeroplane image using the proposed and considered methods of watermarking.

The robustness of the proposed method has been tested by applying different attacks on the watermarked images. In this paper, attacks have been categorized into two classes namely; common signal processing attacks and geometric attacks. The considered common signal processing attacks consists of filtering attacks (mean, median, wiener), noise attacks (gaussian, poisson, salt and pepper), and JPEG compression attacks while rotation, scaling, and cropping are the considered geometric attacks. The attacks are applied on all the eight watermarked images embedded with RTU logo and aeroplane images. The comparison of NC values after applying the common signal processing attacks on watermarked images embedded with RTU logo are depicted in Table 3 while Table 4 shows the NC values for watermarked images embedded with aeroplane image. After applying the geometric attacks, the measured NC values for both the watermark images are compared in Table 5. From Tables 3,4 , and 5 , it is observed that the robustness of watermarked images embedded with RTU logo have higher values of $\mathrm{NC}$ as compared to aeroplane image due to the coarseness of aeroplane image. The comparative results show that the proposed method outperforms other methods for all the considered attacks except JPEG compression and rotation where the method of $\mathrm{Su}$ et al. shows slightly better robustness.

\section{Conclusion}

This paper proposes a novel DWT-based color image watermarking method using UCS and ABC method. The use of uncorrelated color space increases the effective utilization of all color channels of host image which is not feasible in correlated color spaces while ABC is used for optimizing the strength factors to improve the quality and robustness of the proposed method. As per our best knowledge this is the first inline work to use the UCS along with $\mathrm{ABC}$ for effective color image watermarking method. The performance of the proposed method has been measured in terms of quality and robustness against different signal processing attacks and results are compared with the work of Chou and $\mathrm{Wu}^{21}$ and $\mathrm{Su}$ et al. ${ }^{23}$. The results validate that the proposed method is bet- 


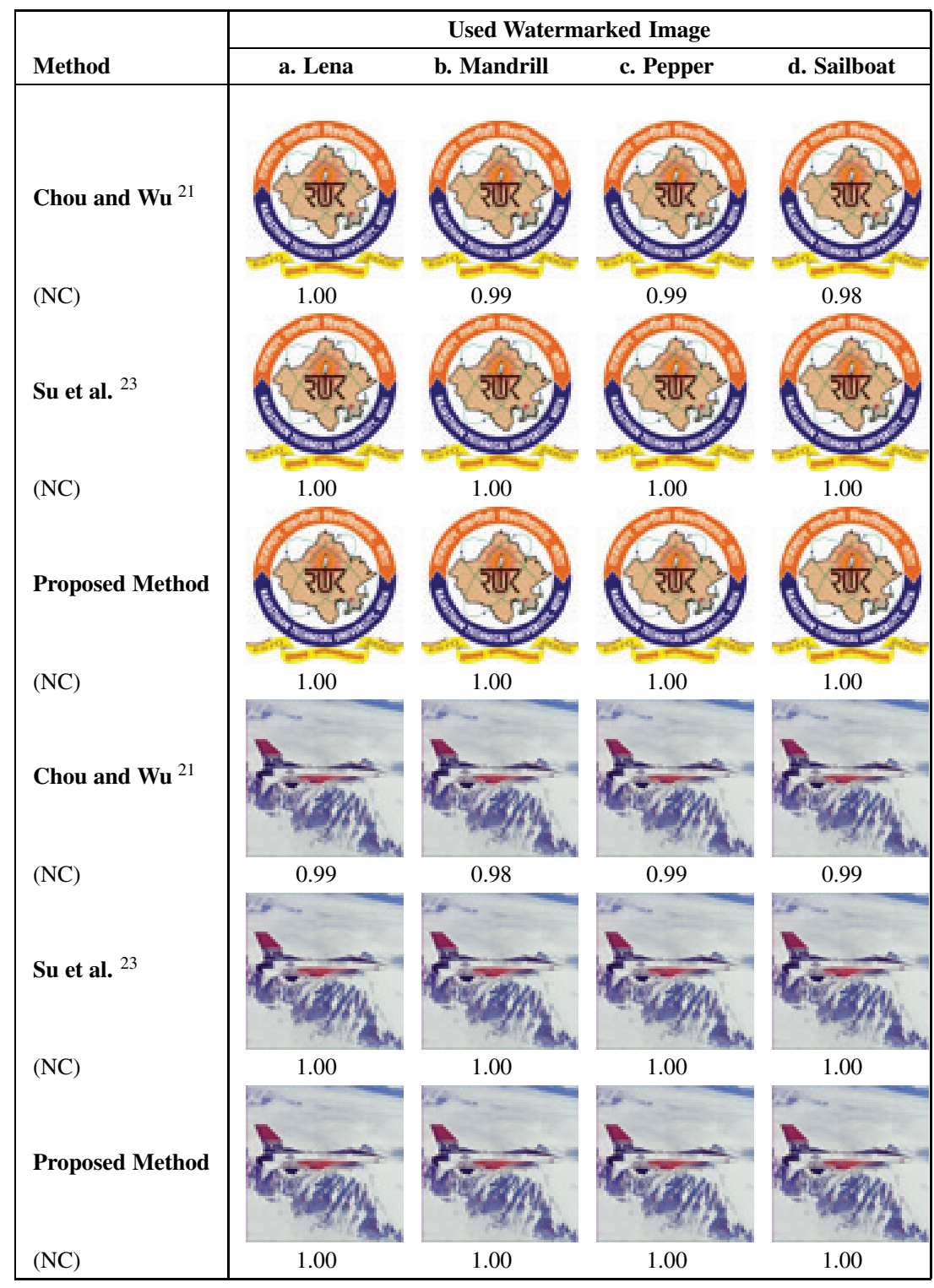

Figure 11: Comparison of extracted watermarks by considered and proposed methods along with their corresponding NC values. Columns shows extracted watermarks from watermarked image namely (a). Lena, (b). Mandrill, (c). Pepper, and (d). Sailboat using the considered and proposed methods mentioned in first column. First three rows shows the extraction of RTU logo while last three shows extraction of aeroplane watermark image. 
Table 2: Average subjective quality comparison of original and watermarked images by 10 human beings in the scale of 0 to 5 .

\begin{tabular}{clllll}
\hline \multirow{2}{*}{ S.No. } & \multirow{2}{*}{$\begin{array}{l}\text { Watermark } \\
\text { image }\end{array}$} & Watermarked image & \multicolumn{3}{c}{ Average Score of 10 Human beings } \\
\cline { 4 - 6 } & & Su et al. ${ }^{23}$ & $\begin{array}{l}\text { Chou and Wu } \\
21\end{array}$ & $\begin{array}{l}\text { Proposed } \\
\text { Method }\end{array}$ \\
\hline 1. & RTU Logo & Lena & 5 & 5 & 5 \\
& & Mandrill & 5 & 5 & 5 \\
& Pepper & 5 & 5 & 5 \\
& Sailboat & 5 & 5 & 5 \\
2. Aeroplane & Lena & 5 & 5 & 5 \\
& Mandrill & 5 & 5 & 5 \\
& & Pepper & 5 & 5 & 5 \\
& Sailboat & 5 & 5 & 5 \\
\hline
\end{tabular}

ter than other methods for all the considered parameters except slight decay in JPEG compression and rotation attacks as compared to Su et al. ${ }^{23}$. Therefore, it is concluded that the proposed method has high quality and robust results and can further be used for protection of the copyrighted images. The present work can be further investigated for other multimedia contents such as video signals. Also a comparative study of GA, PSO and ABC can also be taken up for future research in terms of computational time, complexity, etc.

\section{References}

1. N. Nikolaidis, I. Pitas, Robust image watermarking in the spatial domain, Signal processing, Elsevier, Vol. 66, pp. 385-403, (1998).

2. P. S. Huang, C.-S. Chiang, C. P. Chang, T. M. Tu, Robust spatial watermarking technique for colour images via direct saturation adjustment, IEE ProceedingsVision, Image and Signal Processing, Vol. 152, pp. 561-574, (2005).

3. C. T. Hsu, J. L. Wu, Hidden digital watermarks in images, IEEE Transactions on Image Processing, Vol. 8, pp. 58-68, (1999).

4. G. M. Su, An overview of transparent and robust digital image watermarking (2008).

5. T. H. Lan, A. H. Tewfik, A novel high-capacity dataembedding system, IEEE Transactions on Image Processing, Vol. 15, pp. 2431-2440, (2006).

6. D. Kundur, D. Hatzinakos, Toward robust logo watermarking using multiresolution image fusion principles, IEEE Transactions on Multimedia, Vol. 6, pp. 185-198, (2004).

7. A. A. Reddy, B. N. Chatterji, A new wavelet based logo-watermarking scheme, Pattern Recognition Letters, Elsevier, Vol. 26, pp. 1019-1027, (2005).
8. L. Ghouti, A. Bouridane, M. K. Ibrahim, S. Boussakta, Digital image watermarking using balanced multiwavelets, IEEE Transactions on Signal Processing, Vol. 54, pp. 1519-1536, (2006).

9. E. Vahedi, R. A. Zoroofi, M. Shiva, Toward a new wavelet-based watermarking approach for color images using bio-inspired optimization principles, Digital Signal Processing, Elsevier, Vol. 22, pp. 153-162 (2012).

10. V. Fotopoulos, A. N. Skodras, Digital image watermarking: An overview, Eurasip Newsletter, Vol. 14, pp. 10-19 (2003).

11. C. Y. Lin, M. Wu, J. A. Bloom, I. J. Cox, M. L. Miller, Y. M. Lui, Rotation, scale, and translation resilient public watermarking for images, in: Proc. of Electronic Imaging,International Society for Optics and Photonics, (2000).

12. B. S. Kim, J. G. Choi, C. H. Park, J. U. Won, D. M. Kwak, S. K. Oh, C. R. Koh, K. H. Park, Robust digital image watermarking method against geometrical attacks, Real-Time Imaging, Vol. 9, pp. 139-149, (2003).

13. P. Su, C. C. Kuo, Information embedding in JPEG2000 compressed images, in: in Proc. of IEEE International Symposium on Circuits and Systems, Vol. 3, pp. III-802, (2003).

14. S. Agreste, G. Andaloro, D. Prestipino, L. Puccio, An image adaptive, wavelet-based watermarking of digital images, Journal of Computational and Applied Mathematics, Elsevier, Vol. 210, pp. 13-21, (2007).

15. S. Agreste, G. Andaloro, A new approach to preprocessing digital image for wavelet-based watermark, Journal of Computational and Applied Mathematics, Elsevier, Vol. 221, pp. 274-283, (2008).

16. P. Kumsawat, K. Attakitmongcol, A. Srikaew, An optimal robust digital image watermarking based on genetic algorithms in multiwavelet domain, WSEAS Transactions on Signal Processing, Vol. 5, pp. 42-51, 


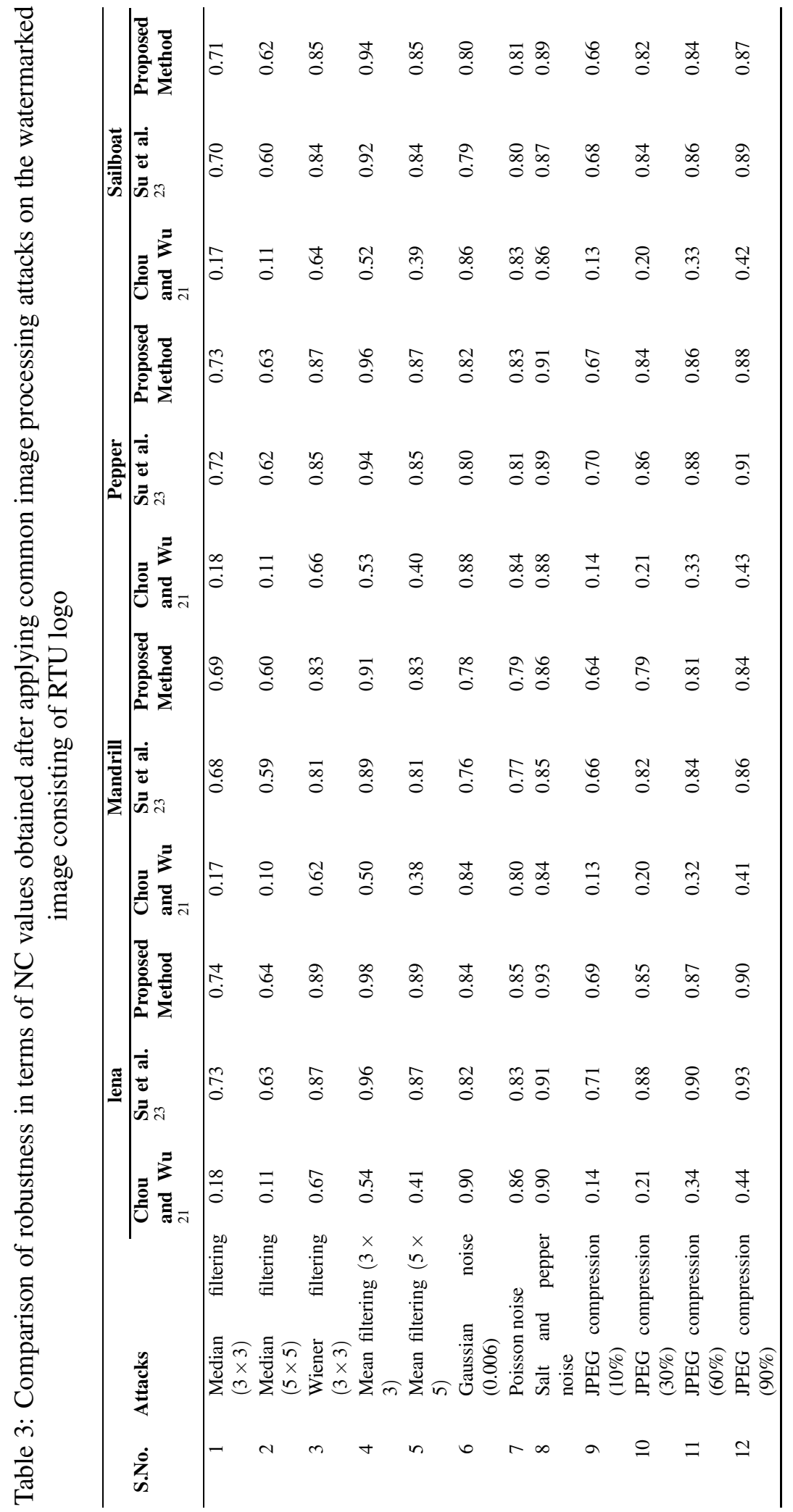




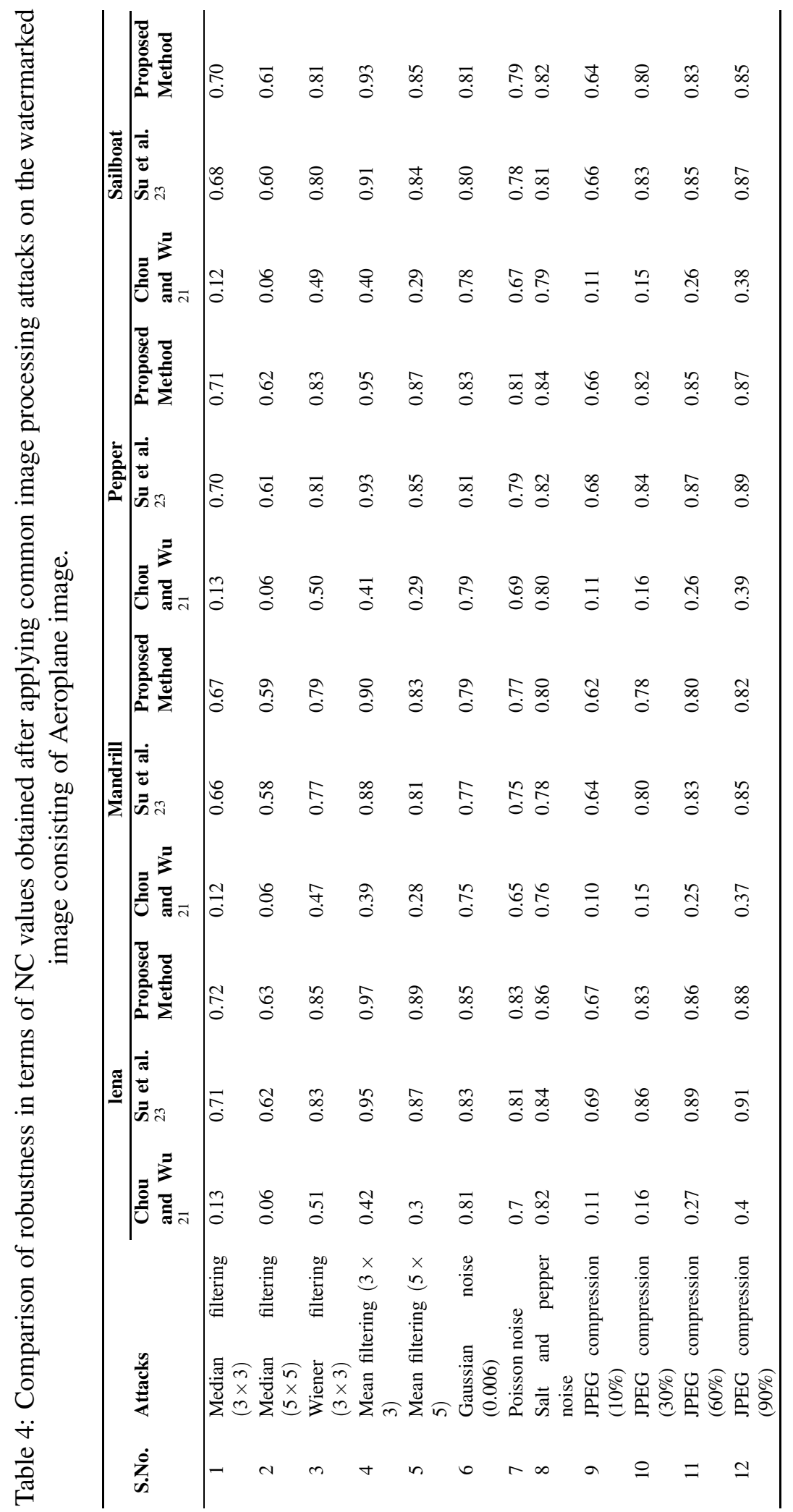




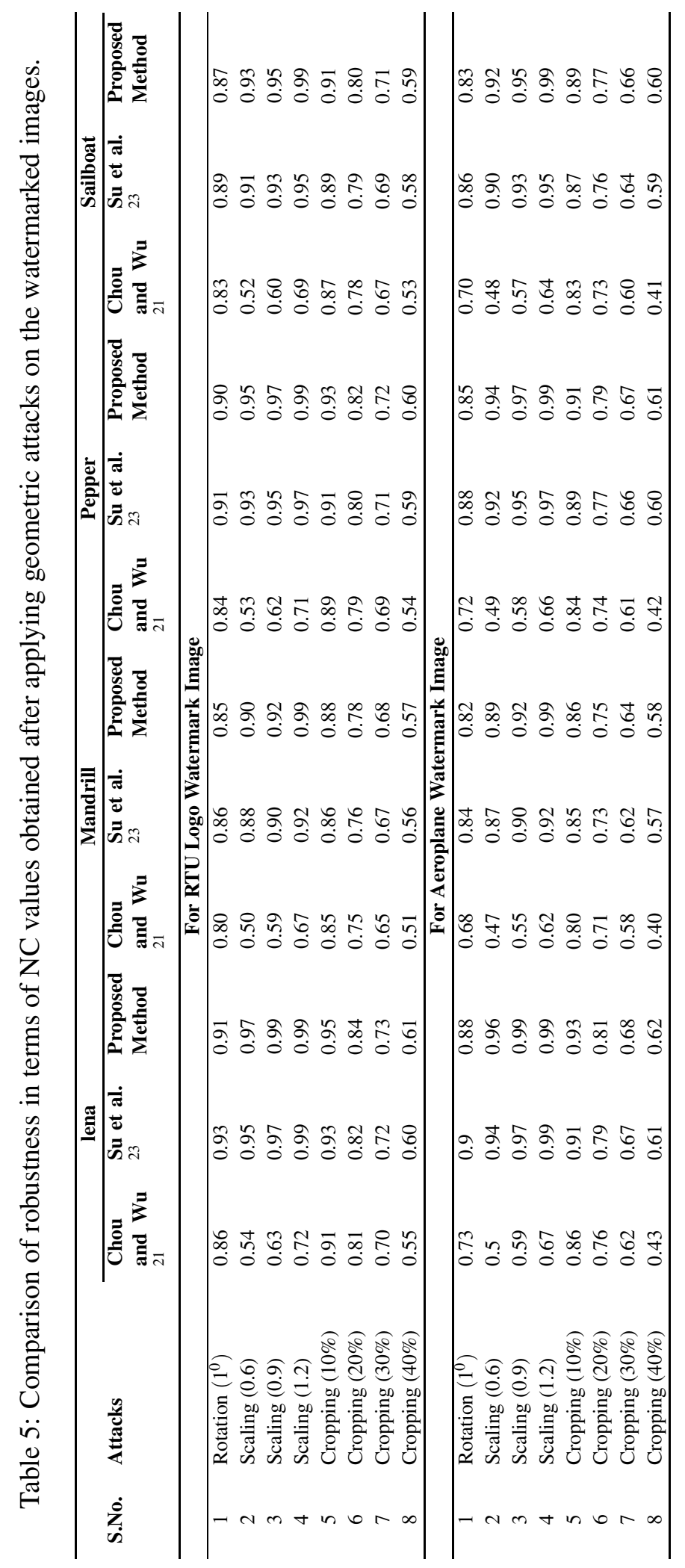


(2009).

17. Z. Wang, X. Sun, D. Zhang, A novel watermarking scheme based on PSO algorithm, in: Proc. of International Conference on Bio-Inspired Computational Intelligence and Applications, Springer, (2007).

18. V. Aslantas, S. Ozer, S. Ozturk, Improving the performance of DCT-based fragile watermarking using intelligent optimization algorithms, Optics Communications, Elsevier, Vol. 282, pp. 2806-2817, (2009) .

19. D. Karaboga, B. Akay, A comparative study of artificial bee colony algorithm, Applied Mathematics and Computation, Elsevier, Vol. 214, pp. 108-132, (2009).

20. E. Vahedi, C. Lucas, R. A. Zoroofi, M. Shiva, A new approach for image watermarking by using particle swarm optimization, in: Proc. of IEEE International Conference on Signal Processing and Communications, (2007).

21. C. H. Chou, T. L. Wu, Embedding color watermarks in color images, EURASIP Journal on Applied Signal Processing, Hindawi Publishing Corp., Vol. 2003, pp. 32-40, (2003).

22. N. H. Golea, R. Seghir, R. Benzid, A blind rgb color image watermarking based on singular value decomposition, in: Proc. of IEEE International Conference on Computer Systems and Applications, (2010).

23. Q. Su, Y. Niu, G. Wang, S. Jia, J. Yue, Color image blind watermarking scheme based on QR decompo- sition, Signal Processing, Elsevier, Vol. 94, pp. 219235, (2014).

24. C. H. Chou, K. C. Liu, A perceptually tuned watermarking scheme for color images, IEEE Transactions on Image Processing, Vol. 19, pp. 2966-2982, (2010).

25. S. Ren, T. Zhang, Study of reversible information hiding scheme based on CARDBAL2 and color field structure, Advanced Materials Research, Trans. Tech. Publ., Vol. 225, pp. 275-279, (2011).

26. Wang, Xiang-yang and Wang, Chun-peng and Yang, Hong-ying and Niu, Pan-pan, A robust blind color image watermarking in quaternion fourier transform domain, Journal of Systems and Software, Elsevier, Vol. 86, pp. 255-277, (2012).

27. P. T. Reinhard E, Colour spaces for colour transfer, in: Proc. of International Conference on Computational Color Imaging, Springer, (2011).

28. M. Saraswat, K. V. Arya, Colour normalisation of histopathological images, Computer Methods in Biomechanics and Biomedical Engineering: Imaging \& Visualization, Taylor \& Francis, Vol. 1, 185-197, (2013).

29. Liu, Chengjun, Learning the uncorrelated, independent, and discriminating color spaces for face recognition, IEEE Transaction on Information Forensics Security, Vol. 3, pp. 213-222, (2008).

30. http://sipi.usc.edu/database/database.php, June, 2014. 